\title{
Management of Bone Disease in Gaucher Disease Type 1: Clinical Practice
}

\author{
Gaetano Giuffrida • Maria Domenica Cappellini • Francesca Carubbi • \\ Maja Di Rocco • Giovanni Iolascon
}

To view enhanced content go to www.advancesintherapy.com

Received: October 15, 2014

(C) Springer Healthcare 2014

\section{ABSTRACT}

Gaucher disease is a rare autosomal recessive disorder of glycosphingolipid metabolism resulting from deficient activity of the lysosomal enzyme beta-glucocerebrosidase

Collaborators: Elena Cassinerio, Fondazione Ca Granda IRCCS, Policlinico Hospital, Milan, Italy; Rita Lombardo, Ernesto Di Francesco, University of Catania, Ospedale Ferrarotto, Catania, Italy; Annalisa Madeo, Gaslini Institute, Genoa, Italy; Chiara Masetti, University of Modena and Reggio Emilia, AUSL Modena, Italy; Antimo Moretti, Second University of Naples, Italy; Maria Ruberto, Second University of Naples, Italy; Maria Francesca Zenobii, University of Modena and Reggio Emilia, AUSL Modena, Italy.

Electronic supplementary material The online version of this article (doi:10.1007/s12325-014-0174-0) contains supplementary material, which is available to authorized users.

\section{G. Giuffrida}

Division of Hematology, Department of Clinical and Molecular Biomedicine, University of Catania,

Ospedale Ferrarotto, Catania, Italy

M. D. Cappellini

Department of Internal Medicine and Community Health, IRCCS Foundation "Ca' Granda" Policlinico Hospital, Milan, Italy

F. Carubbi

Department of Medicine, Endocrinology,

Metabolism and Geriatrics, University of Modena

and Reggio Emilia, AUSL Modena, Modena, Italy that causes accumulation of glucosylceramide in tissue macrophage with damage to hematological, visceral, and skeletal organ systems. Severity and progression may vary independently among these domains, necessitating individualized therapy. Skeletal involvement is highly prevalent and often associated with intense pain, impaired mobility, and reduced quality of life. Enzyme replacement therapy improves parameters in all affected domains, but skeletal involvement requires longer treatment and higher dosages to obtain significant results. Despite numerous papers on bone complications in patients with Gaucher disease, there are no specific indications on how to assess properly bone involvement in such condition, the frequency

\section{Di Rocco}

Unit of Rare Diseases, Department of Pediatrics, Gaslini Institute, Genoa, Italy

G. Iolascon ( $\square)$

Department of Medical and Surgical Specialties and

Dentistry, Second University of Naples, Via de Crecchio, 4, 80138 Naples, Italy e-mail: giovanni.iolascon@gmail.com 
of assessment, the use of markers for osteoblast and osteoclast activity, or the administration of bisphosphonates or other symptomatic drugs in adult and pediatric patients. Starting from a reevaluation of cases with bone involvement, we have identified some common errors in the diagnostic approach and management. The aim of this paper was to propose a methodological and critical approach to the diagnosis, followup and treatment of bone disease in patients with Gaucher disease type 1.

Keywords: Bone; Gaucher disease; Dual energy $\mathrm{X}$-ray absorptiometry; Magnetic resonance imaging; MRI; Skeletal disease

\section{INTRODUCTION}

Gaucher disease (GD; OMIM \#230800) is a rare autosomal recessive hereditary disorder of glycosphingolipid metabolism resulting from deficient activity of the lysosomal enzyme $\quad \beta$-glucocerebrosidase (EC 3.2.1.45; lysosomal glucocerebrosidase). Glucosylceramide accumulates in macrophages (Gaucher cells) in the spleen, liver, and bone marrow. Although GD has a continuous spectrum of severity, it is traditionally classified into three forms: type 1 (chronic; lacking early onset neuronopathy), type 2 (acute; with early onset neuronopathy), and type 3 (chronic; with early onset neuronopathy) [1]. Type 1 GD accounts for the vast majority of cases, with an incidence of approximately $1 / 40-60,000$ in the general population [2,3] and $1 / 500$ in the Ashkenazy population [4].

GD type 1 is a chronic multi-organ disorder with a variety of signs and symptoms that include hepatomegaly, splenomegaly, (pan)cytopenia, pulmonary disease, and various bone manifestations. The severity and clinical course of the disease are extremely heterogeneous, varying between patients and affected domains in the same patient [1]. Bone pathology in GD affects both the marrow and structural compartments, although the precise pathophysiological mechanisms are not yet well understood. Bone marrow infiltration by Gaucher cells may cause vascular occlusion, ischemia, and compression, whereas mineralization defects may result from abnormal bone remodeling [1]. Skeletal manifestations are the most disabling long-term complication of GD, and more than $80 \%$ of patients have signs of bone involvement at diagnosis $[5,6]$. These may include abnormal bone remodeling, osteopenia, lytic lesions, pathological fractures, vertebral collapse, and avascular osteonecrosisparticularly at the femoral or humeral headswith possible bone collapse and joint involvement [7]. Osteopenia is common in patients with GD, and often occurs early in life, resulting in reduced bone mineral density (BMD) with lower peak bone mass and increased risk of complications associated with bone fragility [8].

Skeletal involvement is a compelling indication for enzyme replacement therapy (ERT) and some bone parameters are key treatment goals [8, 9]. While patients with bone pain and bone crisis resulting from bone marrow infiltration generally respond to ERT within 2 years [10], improvement of bone mineral density (BMD) is markedly dosedependent and requires up to 8 years of highdosage therapy to normalize [11]. Some bone manifestations are irreversible [12]; thus, early and sustained treatment is critical. Adequate treatment is especially important during adolescence and early adulthood, when peak bone mass is accrued [13]. Moreover, early treatment may reduce the risk of avascular bone necrosis [14]. Over time, impaired quality of life (QoL) may become refractory to 
therapy due to the occurrence of permanent bone sequelae and the need for joint replacements $[15,16]$.

Guidelines have been elaborated for the diagnosis, treatment and follow-up of patients with GD [17, 18]; however, detailed information on the correct evaluation and interpretation of skeletal involvement are lacking. The aim of this paper was to propose a methodological and critical approach to the diagnosis, follow-up, and treatment of bone disease in patients with GD type 1 .

\section{METHODS}

To address the unmet need for improving the approach to bone disease in GD, a panel of physicians with experience in GD diagnosis and treatment, as well as an expert on bone disease, convened three times to review the literature, discuss clinical cases of GD with various forms and presentations of bone involvement, and formulate recommendations for diagnosing, monitoring, and treating skeletal manifestations of GD. Starting from a re-evaluation of cases with bone involvement, we have identified some common errors in the diagnostic approach and management.

The analysis in this article is based on previously conducted studies and does not involve any new studies of human or animal subjects performed by any of the authors.

\section{CASE REPORTS}

Three cases of GD are presented to illustrate situations encountered in clinical practice; several common pitfalls are highlighted (Supplement 1). Suboptimal outcomes in these clinical cases can be attributed to (a) late diagnosis of GD, (b) the improper use or interpretation of imaging methods for the diagnosis and monitoring of skeletal involvement, or (c) inadequate ERT dosage. Based on these observations we reconsider the most important methods for evaluating bone disease in GD and make critical suggestions for their use and interpretation in light of recent advances in osteoporosis.

\section{DISCUSSION}

\section{Imaging Methods for Assessing Bone Damage}

\section{Dual Energy X-ray Absorptiometry (Table 1)}

The gold standard for quantitative assessment of bone mineral status in adults is dual energy X-ray absorptiometry (DXA), performed at the lumbar spine (L1-L4) and hip (total hip or femoral neck). In children, in addition to DXA performed on the L1-L4 lumbar vertebrae, scans of the total body less head (TBLH) are mandatory, whereas measurement of BMD at the femur is not recommended due to high variability in skeletal maturity [19]. The parameters obtained from these measurements are bone mineral content (BMC) in grams, area in $\mathrm{cm}^{2}$, and BMD in $\mathrm{g} / \mathrm{cm}^{2}$. Because of the twodimensional nature of DXA, measurements are strongly influenced by bone size, skeletal maturity, and pubertal stage. Small bones may lead to an underestimation of BMD. Other confounding factors include avascular necrosis of the proximal femur and vertebral fractures, which may provide misleading results. In adults, the presence of osteophytes, vascular calcifications, and calculi can lead to overestimation of bone mass.

The reference DXA parameter for premenopausal women, for men younger than 
Table 1 Key points for using dual energy X-ray absorptiometry (DXA) for diagnosis and follow-up in patients with Gaucher disease (GD)

\section{Procedure}

The essential parameters are bone mineral content in grams, bone area $\left(\mathrm{cm}^{2}\right)$, and bone mass density (BMD) $\left(\mathrm{g} / \mathrm{cm}^{2}\right)$

Serial measurements of BMD should be performed with the same equipment and, if possible, by the same operator

\section{Sites to examine}

Lumbar spine (L1-L4)

Total body less head (TBLH)

Proximal femur (total hip and femoral neck)

When these sites cannot be measured, the distal third of the radius of the non-dominant forearm is evaluated

\section{Interpretation}

DXA should be interpreted using the Z-score as a reference value. Z-scores below -2.0 indicate reduced bone mineral density. In postmenopausal women and in men aged $\geq 50$ years the T-score is interpreted using the World Health Organization (WHO) classification for osteopenia/osteoporosis, considering methodological precision and least significant change detectable

\section{Frequency}

DXA should be performed at baseline and after 12 months in untreated asymptomatic patients and in patients who have not reached therapeutic goals on enzyme replacement therapy (ERT). When therapeutic goals are achieved, the guidelines recommend follow-up every 12-24 months. However, in our experience this can be carried out every 36 months, in the absence of inter-current conditions associated with BMD loss

\section{Recommendations for pediatric patients}

DXA is performed in patients aged $\geq 5$ years with scans of the lumbar vertebrae (L1-L4) and TBLH; measurement of $\mathrm{BMD}$ at the femur is not recommended. Results are interpreted with the $\mathrm{Z}$-score

50 years old, and for the pediatric population is the Z-score, which refers to the mean BMD of the subject compared to a population of similar age, sex, and ethnicity [20]. In the aforementioned populations, DXA measurements with a Z-score less than or equal to -2.0 should be reported as "BMC or BMD below the expected range for chronological age" and not with the term "osteopenia" [21], whereas the diagnosis of osteoporosis should not be based only on BMD values. For postmenopausal women and men aged $\geq 50$ years, the preferred parameter is the T-score, which expresses BMD compared to that of young adults (aged 20-29 years) of the same sex. It should be interpreted according to the World Health Organization densitometric classification, in which a T-score between -1.0 and -2.5 indicates osteopenia, while a T-score less than -2.5 indicates osteoporosis.

How to Perform DXA Measurement of BMD at the lumbar spine should be performed in the anteroposterior projection, considering the mean BMD of at least two vertebrae among L1-L4, excluding any vertebrae with defects or artifacts. The femoral neck or total hip is the optimal site for fracture risk assessment [22]. In certain circumstances (e.g., for very obese patients or the presence of bone damage in 
vertebral or femoral sites), a different skeletal site may be used, such as the distal third of the radius in the non-dominant arm. The minimum age for densitometric examinations is 5 years.

Regarding methodological variability and the accuracy of measurements, the precision error and least significant change (LSC) detectable must be determined for each DXA apparatus, independently from the information provided by the manufacturer. Strict adherence to protocols is required to obtain accurate results. For complete instructions, refer to appendix II of the International Society for Clinical Densitometry (ISCD) 2013 position document [20]. Equipment accuracy must be assessed on a representative sample of patients at any clinic. To analyze precision, 3 measurements must be performed on each of at least 15 patients or 2 measurements on 30 patients, repositioning the patient after each scan. The minimum acceptable precision varies depending on the anatomical site evaluated, in particular, for the lumbar spine it is 9\%, corresponding to an LSC of $5.3 \%$. This margin of error should be taken into account for repeated measurements performed to assess the effectiveness of drug treatment. For example, a $4 \%$ variation in BMD of the lumbar spine after a year of therapy would be below the LSC for this segment (5.3\%). Therefore, the increase in density would not be attributable with certainty to a pharmacological effect. Serial evaluations should consider not only the Z-score (or T-score) and BMD but also the BMC and the area.

\section{Frequency of DXA Follow-Up Current} recommendations indicate that DXA should be performed at baseline and after 12-24 months in untreated patients with GD and in those who have reached therapeutic
Table 2 Key points for the use of MRI to assess bone marrow infiltration (BMI) in the diagnosis and follow-up of patients with GD

\section{Procedure and interpretation}

T1-weighted MRI can measure the marrow fat component; sequences of the femur and spine are used for the quantification of bone marrow infiltration

T2-weighted sequences are used to identify bone infarcts, osteonecrosis, and the differential diagnosis of bone crises. Evaluation of avascular osteonecrosis requires T2-weighted MRI sequences and short-tau inversion recovery (STIR)

The same semi-quantitative assessment method should be used at baseline and follow-up

\section{Frequency}

Current recommendations indicate that MRI should be performed at baseline and repeated every

12-24 months in treated patients with normal scores

24-36 months in untreated patients with normalized scores

12 months in patients with severe bone marrow burden (BMB) scores

24 months for patients with moderate BMB scores

Time dosage is adjusted when significant complications occur

However, we suggest that follow-up may be performed every

12 months if the normalized score is $>1$

24 months if the normalized score is $\leq 1$

36 months in stable patients with good metabolic control and normalized scores consistently $\leq 1$

Time dosage is adjusted, or significant complications occur

\section{Recommendations for pediatric patients}

Age-related bone marrow conversion in children interferes with evaluation of infiltration; currently there are no guidelines or standardized methods for analyzing this parameter 
goals on ERT, whereas patients who have not reached therapeutic goals should be assessed every 12 months [17]. However, given the slow rate of BMD changes we suggest that assessment can be performed every 24-36 months in untreated patients, and every 36 months in patients who have achieved goals for skeletal involvement on ERT. DXA should be repeated at shorter intervals (e.g., 12-24 months) in patients with conditions associated with a rapid loss of bone mass (e.g., corticosteroids therapy). The minimum time interval for evaluating bone density during the course of treatment is 6 months. Serial DXA examinations should be performed using the same device, possibly by the same operator [23].

\section{Magnetic Resonance Imaging (MRI) (Table 2)}

Magnetic resonance imaging (MRI) is an extremely sensitive method for monitoring bone pathology in GD. Protocols include T1weighted spin echo and turbo sequences, short tau inversion recovery (STIR) sequences, as well as T2-weighted spin echo, turbo spin echo or gradient echo sequences. Spin echo MRI sequences are useful for identifying bone marrow by its high fat content, which appears as a hyperintense T1-weighted signal and an intermediate-to-hyperintense T2-weighted signal. Bone marrow infiltration (BMI) by Gaucher cells displaces fatty marrow, resulting in a less intense signal. Regression of BMI, for example as a consequence of ERT, increases marrow fat content and causes the signal intensity to normalize. Some changes are irreversible, such as bone infarction and fibrosis.

MRI is also the most sensitive method to detect osteonecrosis. Increased signal intensity in T1- and T2-weighted images or hyperintense signal in STIR is suggestive of active bone marrow events. STIR may reveal osteonecrosis that is obscured by Gaucher cell infiltration in T1-and T2-weighted images.

The age-related conversion of hematopoietic to fatty bone marrow that occurs in children interferes with evaluation of BMI, and currently there are no guidelines or standardized methods for analyzing this parameter. Therefore, in clinical practice BMI is not assessed in pediatric patients. However, MRI is useful for assessing osteonecrosis and active bone marrow events in pediatric patients.

MRI is a qualitative method, but semiquantitative methods have been developed to assess BMI by Gaucher cells. BMI is clinical relevant because it is an index of disease severity and of the risk of bone crisis and osteonecrosis. Semi-quantitative MRI methods compare signal intensities to an internal reference standard. Six semi-quantitative methods have been developed that differ mainly by the body area evaluated. These methods have been validated by comparison to other methods, and interrater reliability has been assessed at multiple centers [24-29]. Of these, the bone marrow burden (BMB) seems to be the most commonly used method.

A quantitative MRI method known as quantitative chemical shift imaging (QCSI) has also been developed [30]. QCSI quantifies fat content using differences in the resonant frequencies of fat and water in bone marrow. However, it is not universally available, relies on specialist technical input, is time consuming, and prone to both measurement errors and artifacts. For all these reasons, it is not used in clinical practice. BMI can be measured using scintigraphic imaging with semi-quantitative assessment; however, whereas it is widely available, this method has low specificity, and physiological excretion of markers hampers assessment of the abdominal region. Recently, Di Rocco et al. [3] have normalized these methods so that their 
Table 3 Equivalence of disease severity scores for magnetic resonance and scintigraphic evaluation of bone marrow involvement in patients with Gaucher disease

\begin{tabular}{llllll}
\hline Normalized score & Rosenthal & Dusseldorf & Terk & VDR & BMB \\
\hline 0 & 0 & 0 & 0 & $>2.0$ & $0-2$ \\
1 & $1-3$ & $1-3$ & $1^{\mathrm{a}, \mathrm{b}}$ & $1.5-2.0$ & $3-7$ \\
2 & $4-7$ & $4-6$ & $2^{\mathrm{a}, \mathrm{b}}$ & $1.0-1.5$ & $8-12$ \\
3 & $8-11$ & $7-8$ & $3^{\mathrm{a}, \mathrm{b}}$ & $<1.0$ & $13-16$ \\
\hline Normalized score & QCSI fat fraction & S-MRI & $\mathbf{9 9 m T c - s e s t a m i b i}$ & $\mathbf{9 9 m T c - r a d i o c o l l o i d}$ \\
\hline 0 & $>0.30$ & $0-4$ & $0-2$ & Normal \\
1 & $0.30-0.25$ & $5-10$ & $3-4$ & Mild & \\
2 & $0.20-0.25$ & $11-17$ & $5-6$ & Moderate & \\
3 & $<0.20$ & $18-24$ & $7-8$ & Severe &
\end{tabular}

Reproduced with permission from [3]

a The presence of osteonecrosis is not considered in the bone marrow score per se, since it indicates involvement of the bone mineral component rather than the marrow component, which is the primary focus of MRI

${ }^{b}$ Degree of impairment in the normal scintigraphic pattern. BMB bone marrow burden, S-MRI Spanish magnetic resonance index, $V D R$ vertebral disk ratio, $Q C S I$ quantitative chemical shift index

results are now comparable, allowing clinicians and radiologists to use any one of these methods (Table 3).

Frequency of MRI Follow-Up The International Registry Collaborative Gaucher Group (ICGG) has developed recommendations for effective monitoring of adult patients with GD type 1 [17]. These indicate that MRI should be performed at diagnosis and then every 12-24 months for patients not receiving ERT and for those who have not achieved therapeutic goals while receiving ERT, whereas patients who have achieved therapeutic goals while receiving ERT should be monitored every 24-36 months. Assessments should be performed also when changing therapy or in the event of significant clinical complications. Therapeutic goals for BMI have not been established.

Follow-up imaging may be less frequent in untreated patients with minimal disease who lack evidence of progression over several years, or in treated patients with negligible BMI and evidence of stable disease. Conversely, evaluations should be more frequent in untreated symptomatic patients with significant disease burden [17].

MRI is cost-effective in terms of diagnostic value and the cost of therapy; however, it is expensive and for this reason, we propose the following protocol. At baseline, total body semiquantitative MRI should be used to check for osteonecrosis in sites other than the femoral head [31]. The same semi-quantitative method should be used for follow-up. However, in our opinion, total body MRI is not useful for followup. Instead, we suggest performing MRI on specific symptomatic regions.

If the baseline normalized score (see Table 2) is $\leq 1$, MRI should be performed every 24 months; if it is $>1$, MRI should be performed every 12 months. In stable patients with good metabolic control and scores 
consistently $\leq 1$, MRI can be performed every 36 months.

\section{Plain Radiography}

Radiographic examination is widely available and inexpensive, but it has low sensitivity in detecting initial and small changes in skeletal involvement in GD patients [7]. However, it is useful for detecting bone complications such as fractures. In addition, radiographic imaging may reveal skeletal lesions, cortical bone thinning, and Erlenmeyer flask deformation that may lead to incidental diagnosis of GD in asymptomatic patients.

The guideline developed by the International Collaborative Gaucher Group (ICGG) recommends that the initial assessment include X-ray examination of the femora, spine, and any other symptomatic sites, but indicates that MRI provides much more information on BMB [17]. Conventional radiography may be useful for characterizing osteonecrosis of the proximal femur and osteosclerotic areas, when used in the context of an appropriate classification system.

Steinberg has proposed a classification system that we agreed is useful for identifying progression of radiographic damage and providing information to guide surgical approaches (Steinberg et al. 1998). It is derived from the system of Arlet and Ficat [32] and includes additional stages (Table 4).

\section{Biomarkers for Bone Damage}

Bone manifestations commonly involve decreases or increases in the rate of bone turnover and imbalance between bone resorption and formation. Bone turnover can be measured with specific biochemical markers
Table 4 Steinberg (or University of Pennsylvania) classification system for avascular necrosis of the femoral head

Stage Criteria

0 Normal or nondiagnostic radiograph, bone scan, MRI

I Normal radiographs; abnormal bone scan and/or MRI
A: Mild ( $<15 \%$ of femoral head affected)
B: Moderate (15-30\%)
C: Severe $(>30 \%)$

II Cystic and sclerotic changes in femoral head

A: Mild ( $<15 \%$ of femoral head affected)

B: Moderate (15-30\%)

C: Severe $(>30 \%)$

III Subchondral collapse (crescent sign) without flattening

A: Mild ( $<15 \%$ of articular surface)

B: Moderate (15-30\%)

C: Severe $(>30 \%)$

IV Flattening of femoral head

A: Mild $(<15 \%$ of surface and $<2 \mathrm{~mm}$ depression)

B: Moderate $(15-30 \%$ of surface and $2-4 \mathrm{~mm}$ depression)

C: Severe $(>30 \%$ of surface and $>4 \mathrm{~mm}$ depression)

$\mathrm{V} \quad$ Joint narrowing or acetabular changes

A: Mild $^{a}$

B: Moderate

C: Severe ${ }^{a}$

VI Advanced degenerative changes

a Mean femoral head involvement, as determined in stage IV, and estimated acetabular involvement. MRI magnetic resonance imaging. Reproduced with permission from [60] 
Table 5 Biomarkers for bone damage

We suggest that the following markers be measured every 12 months

Serum calcium and phosphorus

Urinary calcium and phosphorus

The bone isoenzyme of alkaline phosphatase (BALP)

Parathyroid hormone

25-Hydroxyvitamin D

In addition, vitamin $\mathrm{D}$ status should be determined and corrected if deficient

for enzyme activities, the release of various structural components of bone, and proteins involved in the processes of bone formation (Table 5). Over the years, most of these parameters have been tested in patients with GD [33-40]. Urinary total hydroxyproline levels are used to identify osteoclastic hyperactivity. Stowens et al. [41] reported a reduction in urinary hydroxyproline levels in about one-third of patients with GD compared with controls, suggesting a reduction in bone resorption in these patients. Furthermore, osteoblast activity also appears to be suppressed in patients with GD not treated with replacement therapy, as demonstrated by a threefold decrease in levels of markers of osteoblast activity: osteocalcin [34] and carboxy-terminal propeptide of procollagen type 1 (P1NP) [35]. More recently, Van Dussen et al [40]. reported a significant reduction in osteocalcin levels, without significant changes in levels of carboxy-terminal telopeptide of type I collagen (CTX, a marker of osteoclast activity), in $50 \%$ of patients with GD type 1 , suggesting that the altered bone turnover is mainly characterized by a reduction of bone formation. Moreover, Mikosch et al. [38] had previously reported that levels of tartrate-resistant acid phosphatase (TRAP), a widely used osteoclast marker, are unchanged in patients with GD.
Recently, a systematic review has consolidated the view that there are many contradictory results in the literature and that currently there is no scientific evidence to support the use of biochemical markers for the early diagnosis or follow-up of bone involvement in GD [42]. CTX and P1NP have gained attention recently; however, there is not sufficient evidence to warrant their use in clinical practice. It is not clear that any of these markers provide information on bone involvement beyond that obtained with the routinely measured indicators of bone metabolism. Furthermore, biochemical markers provide only an instantaneous measurement of status, whereas imaging reflects the cumulative effects of bone gain and loss throughout life. Thus, we recommend periodic measurement of serum calcium and phosphorus, bone isoenzyme of alkaline phosphatase (BALP), PTH, urinary calcium and phosphorus, and 25-hydroxyvitamin D. In addition, because vitamin $\mathrm{D}$ deficiency is common, its status should be determined and corrected if needed.

\section{Enzyme Replacement Therapy}

The availability of enzyme replacement therapy (ERT) has significantly improved the treatment of GD, making it possible to establish meaningful therapeutic goals for skeletal manifestations of GD (Table 6). Three recombinant mannoseterminated forms of glucocerebrosidase are available for ERT: imiglucerase [43] was approved in 1994, velaglucerase alfa [44] was approved in the US and Europe in 2010, and taliglucerase alfa [45] was approved in the US and Israel in 2012, and subsequently in Brazil, Chile, and Mexico. Most of the data on skeletal treatment goals come from studies conducted with imiglucerase, which has been shown to significantly improve all bone parameters identified as therapy goals (Supplementary 
Table 6 Key points for enzyme replacement therapy for bone manifestations

Enzyme replacement therapy (ERT) is the standard of care for Gaucher disease (GD) types 1 and type 3

Dosage is individualized and based on disease severity and the presence of additional risk factors for bone disease progression

Bone manifestations require long-term treatment at higher dosage to achieve a therapeutic effect

Patients with significant skeletal involvement or other risk factors should receive high-dose therapy (60 U/ $\mathrm{kg} /$ every 2 weeks)

Young patients should receive high-dose therapy to promote attainment of adequate peak bone mineral density during this critical period

Tables 1 and 2) and thus may prevent serious skeletal complications such as bone collapse and fractures. Moreover, post-marketing pharmacosurveliance of more than 4,500 patients reveals that imiglucerase is associated with a stable and low rate of adverse events [46].

ERT improves the visceral aspects of GD in the first year, whereas skeletal involvement requires a longer treatment period [10]. Improvements in bone marrow infiltration are seen within the first 3 years of treatment with ERT [25], and better results are achieved at higher dosages [47]. A meta-analysis of clinical studies evaluated the effects of ERT on bone complications from GD, suggesting that the bone marrow fat fraction increases significantly following treatment and that $70 \%$ of adults with GD respond to treatment [48]. ERT increases the hyperintense T1-weighted MRI signal in bone marrow and significantly reduces BMB scores in patients with GD [28, 49].

On the other hand, normalization of BMD requires long-term treatment for up to 8 years, and there is a clear dose-response relationship $[11,50]$. Appropriate dosage is based on disease severity and the risk of progression. Patients are considered to have high-risk disease if they have symptomatic skeletal involvement, significant visceral or hematological involvement, or, in the case of pediatric patients, if they exhibit growth failure [51]. The patient in Case 2 (Supplement) had a low BMD for his age (T-score -3.16) and had experienced a vertebral collapse. BMD did not respond to initial low-dose imiglucerase therapy; however, increasing the dosage to $60 \mathrm{U} / \mathrm{kg}$ every 2 weeks was associated with gradual normalization of BMD.

Data on 889 patients in the International Collaborative Gaucher Group (ICGG) registry revealed low BMD for chronological age among patients in all age groups, with the highest prevalence among adolescents [8]. ERT improved bone mass in all age groups, but the greatest effect was seen in younger subjects, emphasizing that a therapeutic window of opportunity exists and that high-dosage therapy may have the greatest effect in younger patients during the period when peak bone mineral density is accrued. Starting ERT sooner after diagnosis of GD is associated with better outcomes for avascular necrosis [14] and for bone outcomes in general [8]. However, ERT is also effective at stabilizing or slowing the progression of bone disease in adult patients who are diagnosed or treated late and may not have achieved peak bone density.

In patients with suboptimal bone responses to ERT, differential diagnosis should exclude other diseases that can mimic osteoporosis (e.g., osteomalacia, myelomatosis). It is very important to continue ERT at an optimal dosage in patients with poor or partial bone responses while evaluating concomitant conditions that may be driving bone mineral loss, such as hyperthyroidism, Cushing syndrome, hypogonadism, alcoholism, glucocorticoid therapy, or vitamin D deficiency. 


\section{Other Therapies}

Patients who cannot receive treatment with ERT may benefit from substrate reduction therapy (SRT), which reduces the accumulation of glycosphingolipids by inhibiting the formation of their glucosylceramide precursors. Miglustat is a small molecule glucosylceramide synthase inhibitor licensed for use in adults with mild to moderate GD type 1 . However, it is indicated as second line therapy due to its lower efficacy and tolerability [52, 53]. A second glucosylceramide synthase inhibitor (eliglustat) has shown statistically significant improvement in lumbar spine BMD in phase II trials [54, 55] and was recently approved in the US for use in adult patients with GD type 1.

\section{Supportive Therapies to ERT for Treatment of Bone Manifestations in Gaucher Disease}

Patients with bone mineral deficit may benefit from anti-osteoporotic drugs, in addition to specific therapy (ERT) for GD. The fracture risk assessment tool $\left(\right.$ FRAX $\left.^{\circledR}\right)$ from the World Health Organization quantifies the 10-year fracture risk from skeletal fragility due to primary osteoporosis and identifies a therapeutic threshold beyond which pharmacological treatment is indicated (http://www.shef.ac.uk/

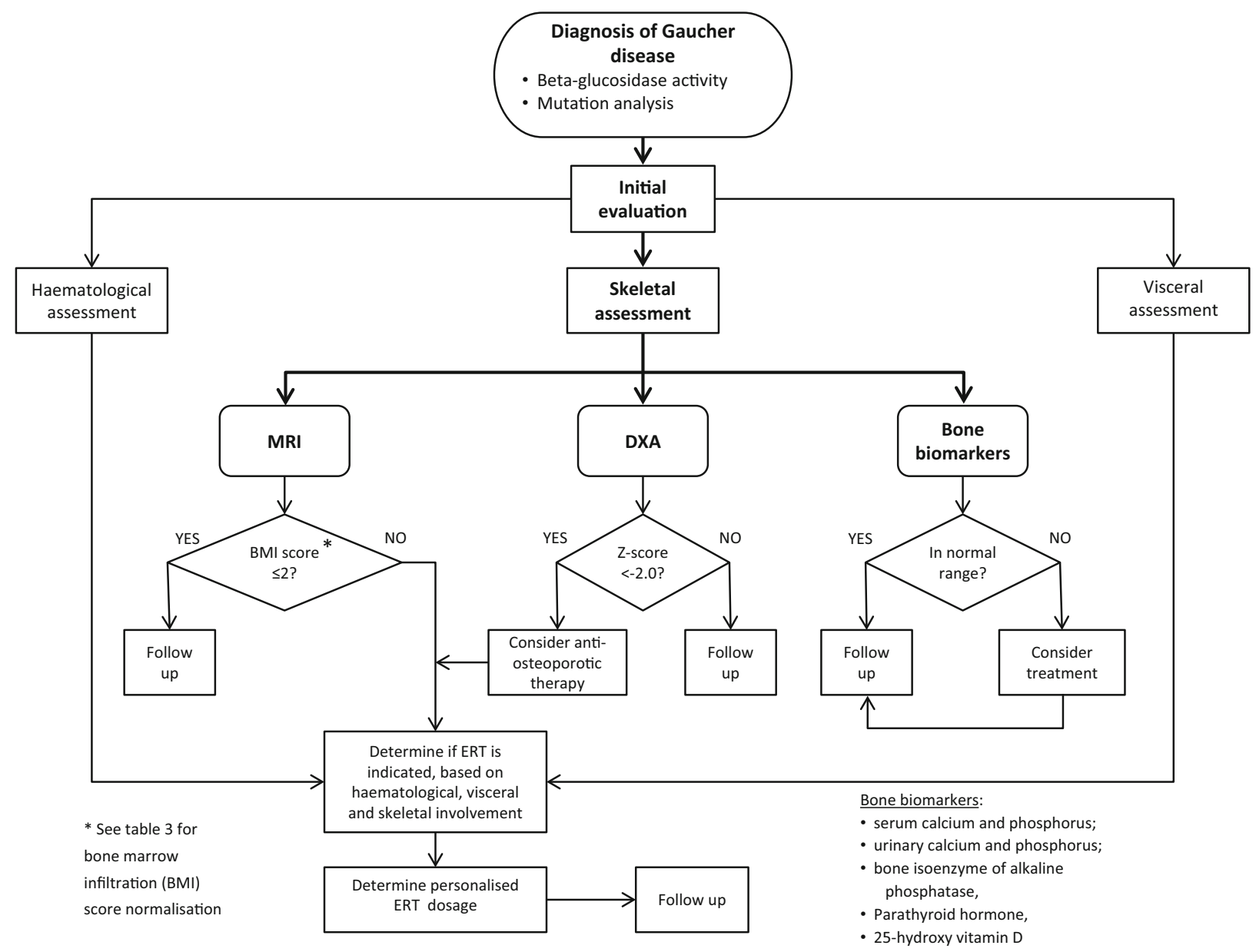

Fig. 1 Proposed algorithm for diagnosing and managing bone manifestations in patients with Gaucher disease. BMI Bone marrow infiltration, $D X A$ dual energy $\mathrm{X}$-ray absorptiometry, ERT enzyme replacement therapy, $M R I$ magnetic resonance imaging 
FRAX/index.aspx). In young subjects, a marked reduction in bone mass documented by DXA is an indication for anti-osteoporotic drugs, particularly bisphosphonates. Treatment with alendronate $40 \mathrm{mg} /$ day provided a significant improvement in BMD at the lumbar spine in patients with GD [56]. Administration of bisphosphonates in GD is supported by guidelines from several scientific societies, though not as preventive therapy [57].

\section{Algorithm for Diagnosis and Follow-Up of Bone Disease in Gaucher Disease Type 1}

The flow charts in Figs. 1 and 2 show proposed algorithms for managing bone disease in GD type 1 , based on the information and discussion in the preceding sections. Figure 1 depicts initial steps for diagnosis and treatment of patients, while Fig. 2 shows steps in monitoring for and follow-up of bone involvement.

\section{CONCLUSION}

The severity of skeletal involvement in type 1 GD is variable, but associated with significant pain, disability, and reduced QoL; thus, all patients should be monitored regularly for the onset and progression of skeletal pathology. DXA is the method of choice for measuring

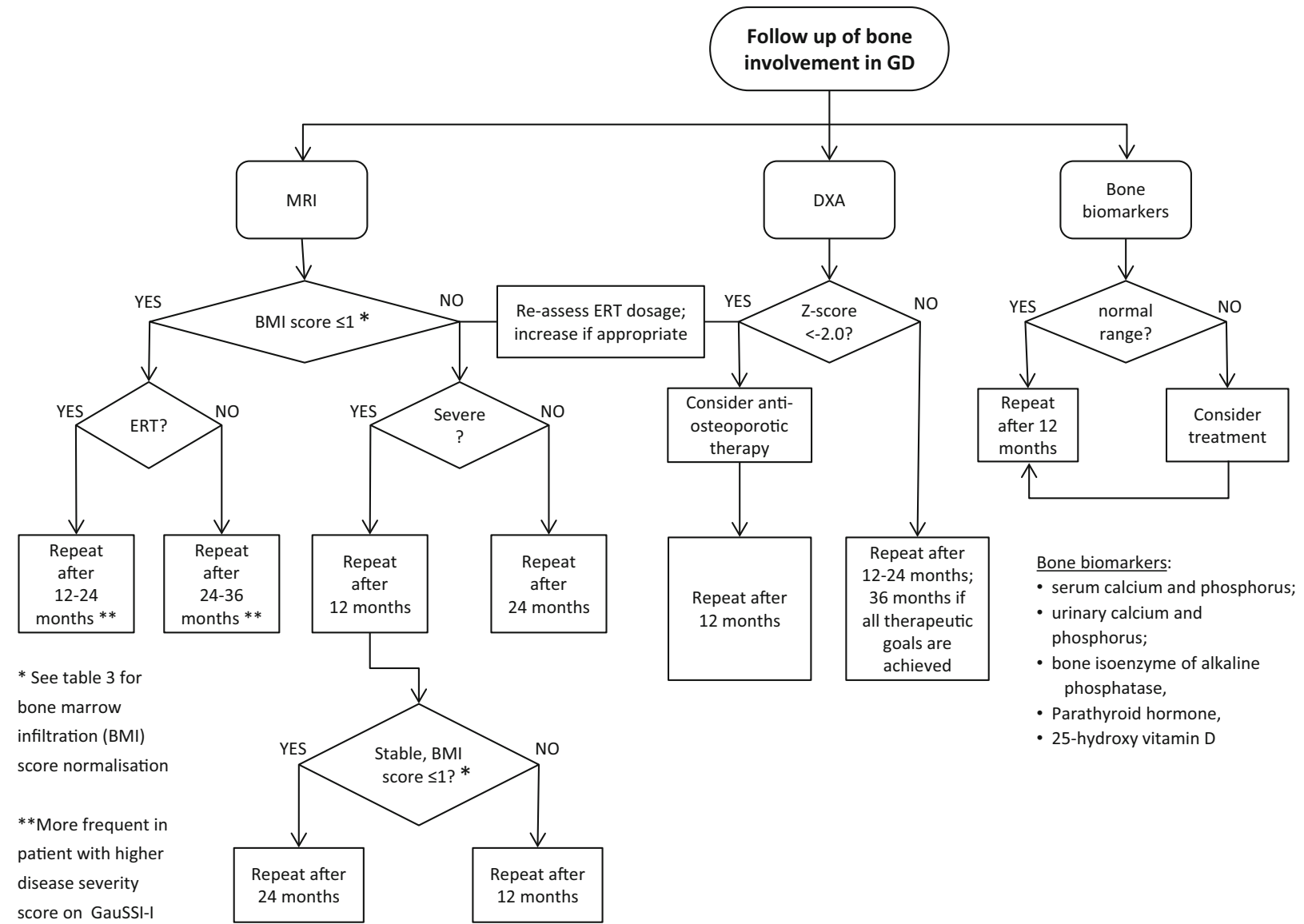

Fig. 2 Proposed algorithm for monitoring, and follow-up of bone involvement in patients with Gaucher disease. BMI Bone marrow infiltration, $D X A$ dual energy $\mathrm{X}$-ray

absorptiometry, ERT enzyme replacement therapy, GauSSI-I Gaucher disease Severity Score Index-type I, $G D$ Gaucher disease, $M R I$ magnetic resonance imaging 
BMD, whereas MRI is the method of choice for evaluating bone marrow infiltration. Correct performance and interpretation of these methods are paramount to obtaining clinically useful information.

ERT has changed the fate of patients with a diagnosis of GD, as demonstrated in numerous clinical studies. Continuous, long-term ERT should be considered for all patients with progressive skeletal involvement and those at risk of developing severe skeletal complications, including children with symptomatic GD. Early treatment at the appropriate individualized dosage is crucial for preventing irreversible skeletal damage. The dosage for individual patients is based on disease severity and response to therapy. Patients at high risk of skeletal disease should receive ERT at a dosage of $60 \mathrm{U} / \mathrm{kg} / 2$ weeks. Anti-osteoporotic therapy should be administered in addition to ERT when indicated.

Looking to the future, therapies combining ERT with SRT have the potential to provide further benefits [58]; however, a small 24-month controlled study did not show a significant benefit for combination therapy with imiglucerase plus miglustat, compared to either drug in monotherapy [59]. Combination therapies should continue to be investigated thoroughly as new agents emerge.

Skeletal manifestations of GD are not necessarily intractable, and patients who are diagnosed and treated early, before irreversible damage occurs, are spared from severe bone involvement. Registry data show that for most adults with late-diagnosed bone involvement, the onset of GD was in childhood. Thus, every effort must be made to monitor for skeletal involvement starting in pediatric patients.

\section{ACKNOWLEDGMENTS}

Sponsorship for this study and article processing charges were funded by Genzyme, a Sanofi company, Modena, Italy. Medical writing assistance for this study was provided by Richard Vernell, an independent medical writer on behalf of Springer Healthcare Communications, Milan, Italy, and funded by Genzyme, a Sanofi company, Modena, Italy. All named authors meet the ICMJE criteria for authorship for this manuscript, take responsibility for the integrity of the work as a whole, and have given final approval for the version to be published.

Author contributions. All authors contributed equally to this work. The authors thank also the collaborators who presented and discussed clinical cases.

Conflict of interest. Gaetano Giuffrida has received honoraria from Genzyme, a Sanofi Company, Modena, Italy; Shire, Florence, Italy; Bayer, Milan, Italy; and Novo Nordisk, Rome, Italy. Maria Domenica Cappellini has received consultancy fees from Novartis, Origgio, Italy; Genzyme, a Sanofi Company, Modena, Italy; and Celgene, Milan, Italy. Francesca Carubbi has received speaker honoraria from Genzyme, a Sanofi Company, Modena, Italy and travel grants from Shire, Florence, Italy and Genzyme, a Sanofi Company, Modena, Italy. Maja Di Rocco has received honoraria from Genzyme, a Sanofi Company, Modena, Italy; Shire, Florence, Italy; Actelion, Imola, Italy; and BioMarin Europe Limited, London, England. Giovanni Iolascon has received honoraria as a speaker from Amgen, Milan, Italy; Grunenthal Italia, Milan, Italy; Eli Lilly Italia, Sesto Fiorentino, Italy; and MSD Italia, Rome, Italy. 
Compliance with ethics guidelines. This article does not contain any studies with human or animal subjects performed by any of the authors.

\section{REFERENCES}

1. Grabowski GA. Phenotype, diagnosis, and treatment of Gaucher's disease. Lancet. 2008;372:1263-71.

2. Meikle PJ, Hopwood JJ, Clague AE, Carey WF. Prevalence of lysosomal storage disorders. JAMA. 1999;281:249-54.

3. Di Rocco M, Giona F, Carubbi F, et al. A new severity score index for phenotypic classification and evaluation of responses to treatment in type I Gaucher disease. Haematologica. 2008;93:1211-8.

4. Zimran A, Gelbart T, Westwood B, Grabowski GA, Beutler E. High frequency of the Gaucher disease mutation at nucleotide 1226 among Ashkenazi Jews. Am J Hum Genet. 1991;49:855-9.

5. Wenstrup RJ, Roca-Espiau M, Weinreb NJ, Bembi B. Skeletal aspects of Gaucher disease: a review. Br J Radiol. 2002;75(Suppl 1):A2-12.

6. Kaplan P, Andersson HC, Kacena KA, Yee JD. The clinical and demographic characteristics of non neuronopathic Gaucher disease in 887 children at diagnosis. Arch Pediatr Adolesc Med. 2006;160:603-8.

7. Vom Dahl S, Poll L, Di Rocco M, et al. Evidencebased recommendations for monitoring bone disease and the response to enzyme replacement therapy in Gaucher patients. Curr Med Res Opin. 2006;22:1045-64.

8. Mistry PK, Weinreb NJ, Kaplan P, Cole JA, Gwosdow AR, Hangartner T. Osteopenia in Gaucher disease develops early in life: response to imiglucerase enzyme therapy in children, adolescents and adults. Blood Cells Mol Dis. 2011;46:66-72.

9. Pastores GM, Weinreb NJ, Aerts $\mathrm{H}$, et al. Therapeutic goals in the treatment of Gaucher disease. Semin Hematol. 2004;41(Suppl 5):4-14.

10. Weinreb NJ, Charrow J, Andersson $\mathrm{HC}$, et al. Effectiveness of enzyme replacement therapy in 1,028 patients with type 1 Gaucher disease after 2-5 years of treatment: a report from the Gaucher Registry. Am J Med. 2002;113:112-9.
11. Wenstrup RJ, Kacena KA, Kaplan P, et al. Effect of enzyme replacement therapy with imiglucerase on BMD in type 1 Gaucher disease. J Bone Miner Res. 2007;22:119-26.

12. Deegan PB, Pavlova E, Tindall J, et al. Osseous manifestations of adult Gaucher disease in the era of enzyme replacement therapy. Medicine (Baltimore). 2011;90:52-60.

13. Andersson H, Kaplan P, Kacena K, et al. Eight-year clinical outcomes of long-term enzyme replacement therapy for 884 children with Gaucher disease type 1. Pediatrics. 2008;122:1182-90.

14. Mistry PK, Deegan P, Vellodi A, et al. Timing of initiation of enzyme replacement therapy after diagnosis of type 1 Gaucher disease: effect on incidence of avascular necrosis. $\mathrm{Br} \mathrm{J}$ Haematol. 2009;147:561-70.

15. Damiano AM, Pastores GM, Ware JE Jr. The healthrelated quality of life of adults with Gaucher's disease receiving enzyme replacement therapy: results from a retrospective study. Qual Life Res. 1998;7:373-86.

16. Giraldo P, Solano V, Pérez-Calvo JI, Giralt M, RubioFélix D, Spanish Group on Gaucher disease. Quality of life related to type 1 Gaucher disease: Spanish experience. Qual Life Res. 2005;14:453-62.

17. Weinreb NJ, Aggio MC, Andersson $\mathrm{HC}$, et al. Gaucher disease type 1: revised recommendations on evaluations and monitoring for adult patients. Semin Hematol. 2004;41(Suppl 5):15-22.

18. Maas M, Hangartner $T$, Mariani $G$, et al. Recommendations for the assessment and monitoring of skeletal manifestations in children with Gaucher disease. Skeletal Radiol. 2008;37:185-8.

19. Bianchi ML, Baim S, Bishop NJ, et al. Official positions of the International Society for Clinical Densitometry (ISCD) on DXA evaluation in children and adolescents. Pediatr Nephrol. 2010;25:37-47.

20. Schousboe JT, Shepherd JA, Bilezikian JP, Baim S. Executive summary of the 2013 International Society for Clinical Densitometry Position Development Conference on bone densitometry. J Clin Densitom. 2013;16:455-66.

21. Gordon CM, Bachrach LK, Carpenter TO, et al. Dual energy X-ray absorptiometry interpretation and reporting in children and adolescents: the 2007 ISCD Pediatric Official Positions. J Clin Densitom. 2008;11:43-58. 
22. Johnell O, Kanis JA, Oden A, et al. Predictive value of BMD for hip and other fractures. J Bone Miner Res. 2005;20:1185-94.

23. Kaplan P, Baris H, De Meirleir L, et al. Revised recommendations for the management of Gaucher disease in children. Eur J Pediatr. 2013;172:447-58.

24. Rosenthal DI, Scott JA, Barranger J, et al. Evaluation of Gaucher disease using magnetic resonance imaging. J Bone Joint Surg Am. 1986;68:802-8.

25. Poll LW, Koch JA, vom Dahl S. Magnetic resonance imaging of bone marrow changes in Gaucher disease during enzyme replacement therapy: first German long-term results. Skeletal Radiol. 2001;30:496-503.

26. Terk MR, Dardashti S, Liebman HA. Bone marrow response in treated patients with Gaucher disease: evaluation by T1-weighted magnetic resonance images and correlation with reduction in liver and spleen volume. Skeletal Radiol. 2000;29:563-71.

27. Vlieger EJ, Maas M, Akkerman EM, Hollak CE, Den Heeten GJ. Vertebra disc ratio as a parameter for bone marrow involvement and its application in Gaucher disease. J Comput Assist Tomogr. 2002;26:843-8.

28. Maas M, van Kuijk C, Stoker J, et al. Quantification of bone involvement in Gaucher disease: MR imaging bone marrow burden score as an alternative to Dixon quantitative chemical shift MR imaging-initial experience. Radiology. 2003;229:554-61.

29. Roca M, Mota J, Alfonso P, Pocoví M, Giraldo P. S-MRI score: a simple method for assessing bone marrow involvement in Gaucher disease. Eur J Radiol. 2007;62:132-7.

30. Maas M, Hollak CE, Akkerman EM, Aerts JM, Stoker J, Den Heeten GJ. Quantification of skeletal involvement in adults with type I Gaucher's disease: fat fraction measured by Dixon quantitative chemical shift imaging as a valid parameter. AJR Am J Roentgenol. 2002;179:961-5.

31. Poll LW, Cox ML, Godehardt E, Steinhof V, vom Dahl S. Whole body MRI in type I Gaucher patients: evaluation of skeletal involvement. Blood Cells Mol Dis. 2011;46:53-9.

32. Ficat RP. Idiopathic bone necrosis of the femoral head. Early diagnosis and treatment. J Bone Joint Surg Br. 1985;67:3-9.

33. Moran MT, Schofield JP, Hayman AR, Shi GP, Young E, Cox TM. Pathologic gene expression in Gaucher disease: up-regulation of cysteine proteinases including osteoclastic cathepsin K. Blood. 2000;96:1969-78.
34. Drugan C, Jebeleanu G, Grigorescu-Sido P, Caillaud C, Craciun AM. Biochemical markers of bone turnover as tools in the evaluation of skeletal involvement in patients with type 1 Gaucher disease. Blood Cells Mol Dis. 2002;28:13-20.

35. Ciana G, Addobbati R, Tamaro G, et al. Gaucher disease and bone: laboratory and skeletal mineral density variations during a long period of enzyme replacement therapy. J Inherit Metab Dis. 2005;28:723-32.

36. Deegan PB, Moran MT, McFarlane I, et al. Clinical evaluation of chemokine and enzymatic biomarkers of Gaucher disease. Blood Cells Mol Dis. 2005;35:259-67.

37. Vellodi A, Foo Y, Cole TJ. Evaluation of three biochemical markers in the monitoring of Gaucher disease. J Inherit Metab Dis. 2005;28:585-92.

38. Mikosch P, Reed M, Baker R, et al. Changes of bone metabolism in seven patients with Gaucher disease treated consecutively with imiglucerase and miglustat. Calcif Tissue Int. 2008;83:43-54.

39. Stirnemann J, Belmatoug N, Vincent C, Fain O, Fantin B, Mentré F. Bone events and evolution of biologic markers in Gaucher disease before and during treatment. Arthritis Res Ther. 2010;12:R156.

40. van Dussen L, Lips P, Everts VE, et al. Markers of bone turnover in Gaucher disease: modeling the evolution of bone disease. J Clin Endocrinol Metab. 2011;96:2194-205.

41. Stowens DW, Teitelbaum SL, Kahn AJ, Barranger JA. Skeletal complications of Gaucher disease. Medicine (Baltimore). 1985;64:310-22.

42. Giuffrida G, Cingari MR, Parrinello N, et al. Bone turnover markers in patients with type 1 Gaucher disease. Hematol Rep. 2012;4:e21.

43. Grabowski GA, Barton NW, Pastores G, et al. Enzyme therapy in type 1 Gaucher disease: comparative efficacy of mannose-terminated glucocerebrosidase from natural and recombinant sources. Ann Intern Med. 1995;122:33-9.

44. Zimran A, Altarescu G, Philips M, et al. Phase $1 / 2$ and extension study of velaglucerasealfa replacement therapy in adults with type 1 Gaucher disease: 48-month experience. Blood. 2010;115:4651-6.

45. Aviezer D, Brill-Almon E, Shaaltiel Y, et al. A plantderived recombinant human glucocerebrosidase enzyme-a preclinical and phase I investigation. PLoS One. 2009;4:e4792. 
46. Starzyk K, Richards S, Yee J, Smith SE, Kingma W. The long-term international safety experience of imiglucerase therapy for Gaucher disease. Mol Genet Metab. 2007;90:157-63.

47. de Fost M, Hollak CE, Groener JE, et al. Superior effects of high-dose enzyme replacement therapy in type 1 Gaucher disease on bone marrow involvement and chitotriosidase levels: a 2-center retrospective analysis. Blood. 2006;108:830-5.

48. Piran S, Amato D. Gaucher disease: a systematic review and meta-analysis of bone complications and their response to treatment. J Inherit Metab Dis. 2010;33:271-9.

49. Robertson PL, Maas M, Goldblatt J. Semiquantitative assessment of skeletal response to enzyme replacement therapy for Gaucher's disease using the bone marrow burden score. AJR Am J Roentgenol. 2007;188:1521-8.

50. Weinreb N, Taylor J, Cox T, Yee J, vom Dahl S. A benchmark analysis of the achievement of therapeutic goals for type 1 Gaucher disease patients treated with imiglucerase. Am J Hematol. 2008;83:890-5.

51. Andersson HC, Charrow J, Kaplan P, et al. Individualization of long-term enzyme replacement therapy for Gaucher disease. Genet Med. 2005;7:105-10.

52. Cox TM, Aerts JM, Andria G, et al. The role of the iminosugar $N$-butyldeoxynojirimycin (miglustat) in the management of type I (non-neuronopathic) Gaucher disease: a position statement. J Inherit Metab Dis. 2003;26:513-26.

53. Hollak CE, Hughes D, van Schaik IN, Schwierin B, Bembi B. Miglustat (Zavesca) in type 1 Gaucher disease: 5-year results of a post-authorisation safety surveillance programme. Pharmacoepidemiol Drug Saf. 2009;18:770-7.

54. Lukina E, Watman N, Arreguin EA, et al. Improvement in hematological, visceral, and skeletal manifestations of Gaucher disease type 1 with oral eliglustat tartrate (Genz-112638) treatment: 2-year results of a phase 2 study. Blood. 2010;116:4095-8.

55. Lukina E, Watman N, Arreguin EA, et al. A phase 2 study of eliglustat tartrate (Genz-112638), an oral substrate reduction therapy for Gaucher disease type 1. Blood. 2010;116:893-9.

56. Wenstrup RJ, Bailey L, Grabowski GA, et al. Gaucher disease: alendronate disodium improves bone mineral density in adults receiving enzyme therapy. Blood. 2004;104:1253-7.
57. Adami S, Bertoldo F, Brandi ML, et al. Guidelines for the diagnosis, prevention and treatment of osteoporosis. Reumatismo. 2009;61:260-84.

58. Cox-Brinkman J, van Breemen MJ, van Maldegem BT, et al. Potential efficacy of enzyme replacement and substrate reduction therapy in three siblings with Gaucher disease type III. J Inherit Metab Dis. 2008;31:745-52.

59. Elstein D, Dweck A, Attias D, et al. Oral maintenance clinical trial with miglustat for type I Gaucher disease: switch from or combination with intravenous enzyme replacement. Blood. 2007;110:2296-301.

60. Steinberg ME, Brighton CT, Corces A. Osteonecrosis of the femoral head; results of core depression and grafting with electrical stimulation. Clin Orthop. 1989;249:199-208.

61. Weinreb NJ, Goldblatt J, Villalobos J, et al. Longterm clinical outcomes in type 1 Gaucher disease following 10 years of imiglucerase treatment. J Inherit Metab Dis. 2013;36:543-53.

62. Sims KB, Pastores GM, Weinreb NJ, et al. Improvement of bone disease by imiglucerase (Cerezyme) therapy in patients with skeletal manifestations of type 1 Gaucher disease: results of a 48-month longitudinal cohort study. Clin Genet. 2008;73:430-40.

63. Charrow J, Dulisse B, Grabowski GA, Weinreb NJ. The effect of enzyme replacement therapy on bone crisis and bone pain in patients with type 1 Gaucher disease. Clin Genet. 2007;71:205-11.

64. Elstein D, Foldes AJ, Zahrieh D, et al. Significant and continuous improvement in bone mineral density among type 1 Gaucher disease patients treated with velaglucerasealfa: 69-month experience, including dose reduction. Blood Cells Mol Dis. 2011;47:56-61.

65. Elstein D, Haims AH, Zahrieh D, Cohn GM, Zimran A. Impact of velaglucerasealfa on bone marrow burden score in adult patients with type 1 Gaucher disease: 7-Year follow-up. Blood Cells Mol Dis. 2014;53:56-60.

66. Weinreb N, Barranger J, Packman S, et al. Imiglucerase (Cerezyme) improves quality of life in patients with skeletal manifestations of Gaucher disease. Clin Genet. 2007;71:576-88.

67. Ida H, Rennert OM, Kobayashi M, Eto Y. Effects of enzyme replacement therapy in thirteen Japanese paediatric patients with Gaucher disease. Eur J Pediatr. 2001;160:21-5. 